\title{
Customers Perspective on Mass-customization of Houses
}

\author{
Krystian Kwiecinski ${ }^{1}$, Jose P. Duarte ${ }^{2}$ \\ ${ }^{1}$ Faculty of Architecture, Warsaw University of Technology, Poland ${ }^{2}$ SCDC, The \\ Pennsylvania State University, USA \\ ${ }^{1}$ krystian.kwiecinski@pw.edu.pl ${ }^{2}$ jxp400@psu.edu
}

\begin{abstract}
This paper presents the results of usability tests of HOPLA (Home Planner), a computer-assisted design system developed to enable customization of house designs. The study aimed to verify whether the proposed method allows non-expert users to configure a house design that meets their expectations in a limited time. The experiments were carried out in two modes of the tool: M mode - modification of a proposed design and $S$ mode - configuration of a design from scratch. The study encompassed two independent experiments carried out on two continents and examined the impact of cultural differences on the expectations of non-expert users towards computer-assisted customization of single-family houses.
\end{abstract}

Keywords: mass-customization, house design, participatory design, usability tests

\section{INTRODUCTION}

Research on the mass-customization of housing gained considerable momentum over the last two decades, due to the use of computational tools. Several approaches have been proposed to address the housing problem and develop design systems to generate solutions that meet user needs (Duarte, 2001; McLeish, 2003; Huang and Krawczyk, 2004; Mohamed, 2013; Khalili-Araghi and Kolarevic, 2016). While most research focused on proposing computer tools that would streamline the customization process, there was less interest in undertaking usability tests. Parallel to advances in research, practice saw the development of online configurators by house builders operating in North America (Living Homes, Blu Homes, Connect Homes, Pennwest Homes, Toll Brothers). Contrary to the tools proposed in research, market tools have mainly focused on presentation and visualization of the "customized" products. In fact, the customization capabilities of such configurators are limited to the selection of finishing materials and appliances. Most available configurators allow users to select from different design models as in the traditional printed catalogues of designs, while only a few support minor alterations to the floorplan or modification of facade elements. This research sought an alternative approach that aimed to verify the possibility for generating alternative design configurations. Proposed interactive design tool for the mass-customization of house layouts was used for assessing the potential of non-expert users' participation in the configuration of house designs, as well as their attitude towards design customization in general. 


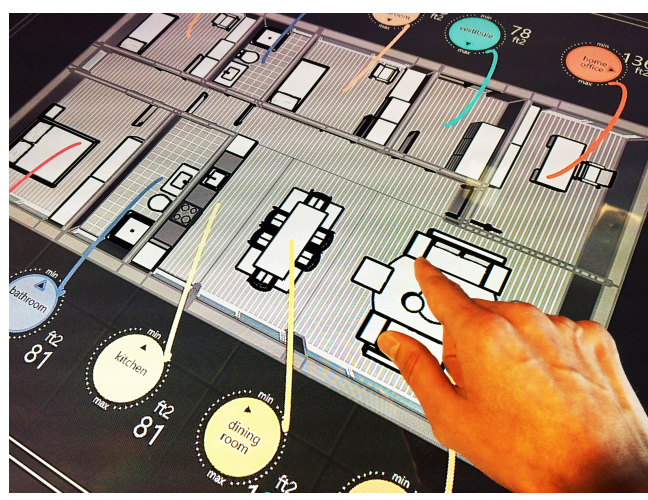

\section{PARTICIPATORY DESIGN TOOL}

For the purpose of this research, HOPLA (Home Planner) a computer-aided design system for the customization of house designs developed by Kwiecinski and Markusiewicz (2018) was adopted. The system consists of two core elements: an algorithm responsible for generating design solutions in response to user input, and an interactive interface allowing users to introduce data and to control the process in an intuitive way. The algorithm is based on a generic grammar, which define meta-design language that can be applied generically in a design domain independently of local specifications (Beirão and Duarte, 2018). Users interact with the system by modifying the position of markers representing each room on a multi-touch screen (Figure 1). The tool allows: to change the layout by modifying the order of rooms on the screen, to change the size of each room by rotating the corresponding marker and to change the outer contour of the building by rotating a dedicated marker. Additionally, rooms can be erased or added by removing or placing the corresponding markers in or out of active spaces on the screen.

The developed design system was designed to enable configuration of design solutions by its users, while ensuring that design solutions meet the encoded design principles. As such, the tool was designed to provide users with freedom to make modi- fications, while limiting design possibilities to ensure compliance with design rules and safety codes at the same time. The designs modified by users when playing with markers on the interactive table are analyzed and then verified by the design system. Based on this analysis, the design system generates a design solution that is presented to the user in the form of an extruded floor plan displayed on a flat screen. If as a consequence of a design move made by the user, the layout does not comply with the formalized design rules, the generative system searches for the configuration that is the closest to the user's input. If the new design solution does not fulfill the user's expectations, it can be further changed by modifying the position of the markers again. The tool allows users to configure house designs in one of two configuration modes: $\mathrm{M}$ mode - modification of the initial design solution and S mode - independent configuration of a solution.

\section{USABILITY STUDIES}

Usability tests of HOPLA with non-expert users (nonarchitects) were carried out in 2018 through two independent experiments, one in the United States at The Pennsylvania State University campus and the other in Poland during the Murator-Expo building fair in Warsaw. The experiment in the US, labelled E02, took four days and had the participation of 30 people and the one in Poland, labelled E03, had the participation of 17 people over two days. The experiment E01 was conducted with architecture students at Warsaw University of Technology and is not considered in this paper. Responses to the questionnaires that participants were asked to fill in after completing the assigned tasks revealed that among participants were people with professional architectural design skills. As a result, the data corresponding to their participation were excluded from the analysis. Eventually, in this study we analyzed data collected from 12 in $\mathrm{M}$ mode and 13 people in S mode, during E02 and from 7 in $\mathrm{M}$ mode and 8 people in $\mathrm{S}$ mode during E03.

During these experiments, participants were
Figure 1

HOPLA interactive user interface using multitouch screen. 
Figure 2

Initial house design

displayed in a

M-mode

configuration. able to independently configure the design of a single-family house using HOPLA in individual sessions. Each session consisted of two parts. In the first part, the participants were handed over the instructions for the design task, according to which they had to configure a floorplan for a single-family house using HOPLA. The house was for a given family of four people, two adults and two children, aged 10 and 14 , and situated on a rectangular plot perpendicular to an access road on the East side, that is, longitudinally along the East-West direction. Participants were asked to consider aspects like the location of rooms in relation to the plot entrance, solar orientation, and the given house position on the plot.

All participants were randomly assigned to one of the two configuration modes: M mode - modification of a design solution and $\mathrm{S}$ mode - independent configuration of a solution. In the M mode, an initial design solution was displayed on the interactive table screen (Figure 2) and the participants' task was to modify the solution. In the S-mode, participants were given an empty workspace and the task was to configure the design from scratch. Each participant had 15 minutes to complete the task.

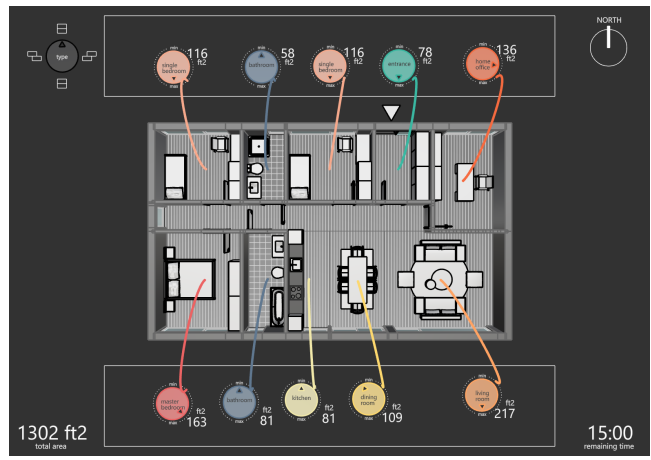

In the second part of the study, each participant was asked to provide feedback by filling in a questionnaire. This consisted of several questions using a 5point Likert scale, designed with the goal of evaluating the usability of the tool, the effectiveness of the design process, and their satisfaction with the final design solution. It also featured open questions regarding general aspects of the developed tool and the proposed participatory design process. The surveys and configured design solutions were made anonymous by assigning them unique numbers.

\section{RESULTS AND DISCUSSION}

In this section, the main observations made during the tests with laypeople are presented, as well as the analysis of the log files obtained during the tests and the results of the questionnaires. The results of the surveys showed that the majority of participants in the experiment were people aged 18-34. They accounted for $88 \%$ and $80 \%$ of the people in the surveys conducted in the USA and in Poland, respectively. The remainder were people aged 35-49 and no person over the age of 50 participated in the study.

The survey included questions about previous experience in designing houses. This question raised the question among some participants if having designed a house in The Sims could be counted as design experience. In these cases, they were always given the same answer, that they should answer what they felt. The collected results showed that $36 \%$ (E02) and $27 \%$ (E03) of participants determined that they had previously designed a house. Almost all of them were aged 18-34, while only one participant over the age of 35 in E02 indicated that he had previously designed a house. This result shows that playing games like The Sims can give users the conviction that they have design experience, thereby signaling the impact of using digital tools simulating the design process on their perception of design experience.

\section{Customized Designs}

All the participants in both experiments finished the given task and configured their houses (Figure 3). Questionnaire answers allowed to analyze the level of the participants' satisfaction with their customized designs. Participants were asked to evaluate the elements of the configured design solutions using a 5point Likert scale. Comparison of the collected answers for the E02 and E03 (Figure 4) shows that par- 

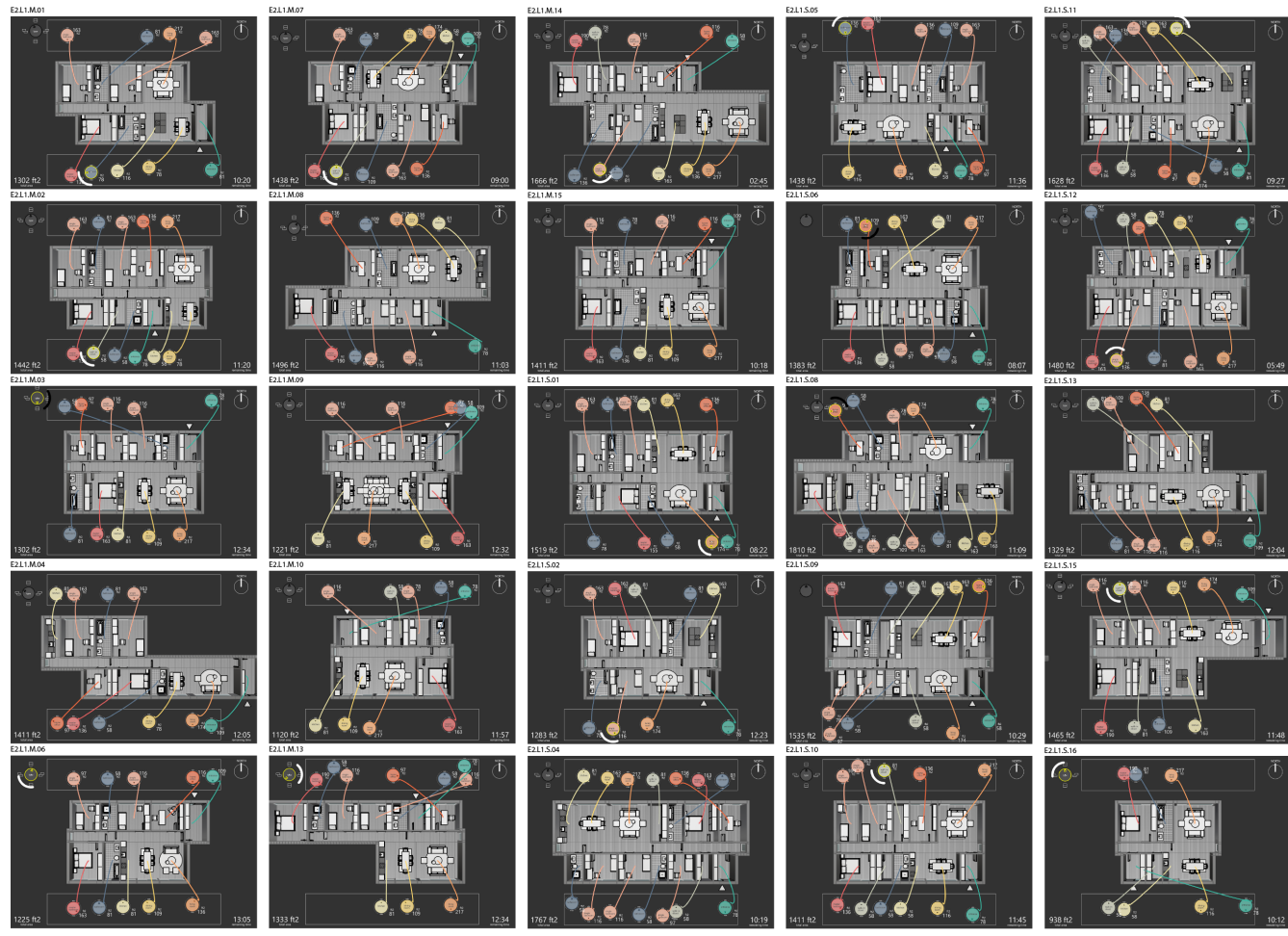

The

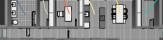

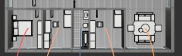

$C_{i} \quad \theta_{i} \theta_{1}$
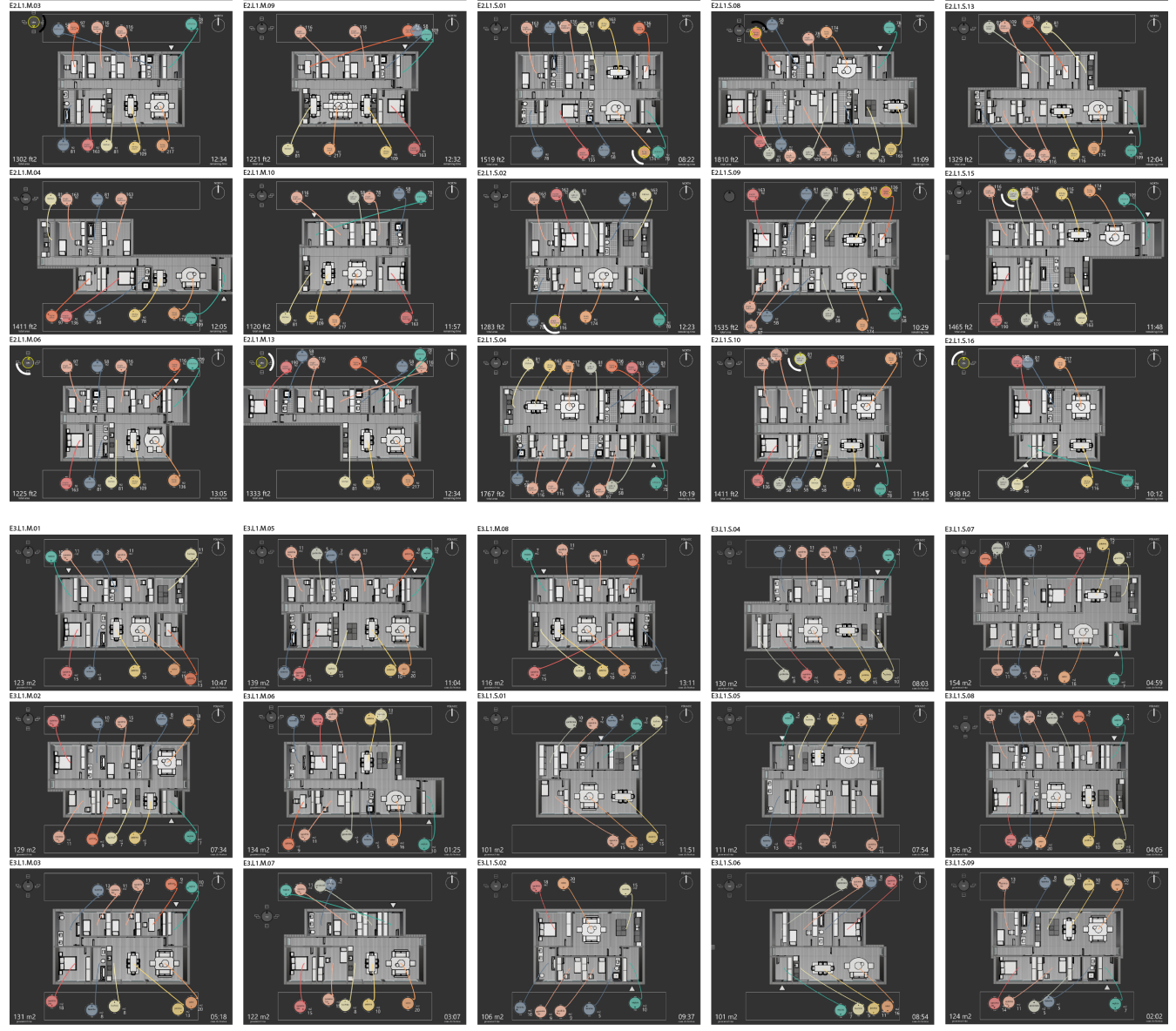

Figure 3

Table of design solutions obtained by participants during the experiments conducted in the USA (E02) and in Poland (E03). 
Figure 4

The result of the survey. Box plots of satisfaction levels with the listed elements of the project [1-very dissatisfied, 5-very satisfied]. On the left, a summary for research conducted in the USA and on the right in Poland.
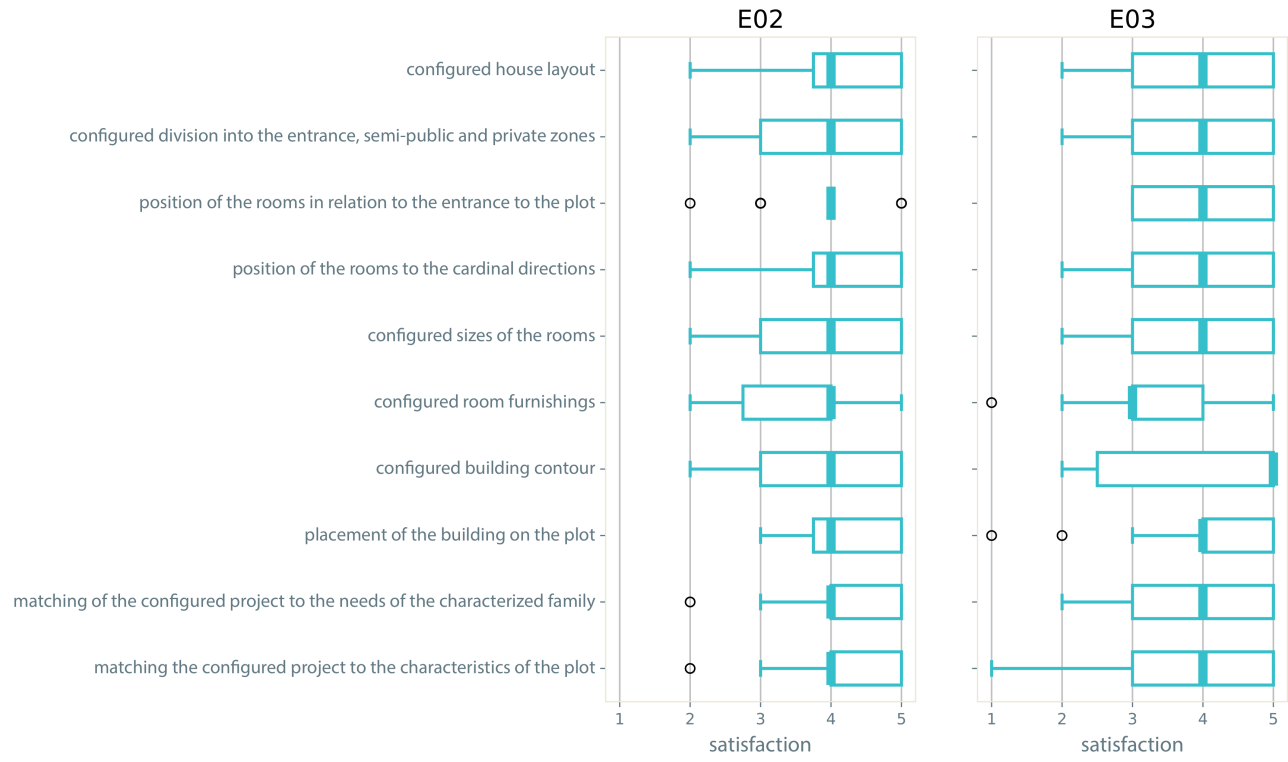

ticipants tended to be very satisfied with their design solution. Their satisfaction with the majority of the elements of the solutions have median equal to 4 out of 5-completely satisfied. The Krustal-Wallis test showed that there is not a statistically significant difference amongst the medians at the 95,0\% confidence level ( $P$-value $=0,956952$ for E02 and $P$ value $=0,548812$ for E03). These findings were confirmed by participants who additionally answered the open question: "Are you satisfied with the configured project solution and why?" where most of them expressed satisfaction with the design and with the functionality of the tool.

\section{Computer Assisted Participatory Design Process}

The data collected during the experiments allowed us to take a closer look at the configuration process of customized solutions using HOPLA. Data regarding the design process was collected in one-second intervals during design sessions. The collected data allowed to analyze the process of computer-assisted co-design in the following categories: time to configure the design solution and change of room configuration.

All participants completed the given design task within the set time of 15 minutes. Additionally, 92\% and $93 \%$ of participants in the E02 and in the E03 experiments, respectively, confirmed in the questionnaire that there was "enough time to configure the design". The collected data allowed to compare all recorded participatory design processes by examining the number of configured rooms over the configuration time (Figure 5). Graphs illustrate the basic difference between the configuration in $\mathrm{M}$ mode, where the user modifies an initial design consisting of 10 rooms, and S mode, where the design has to be created from scratch. In the $\mathrm{M}$ mode, most participants maintained a similar number of markers on the table throughout the entire configuration, except for the experiment E2.L1.M.10 and the experiment E3.L1.M.06. The first one is an example of a configu- 


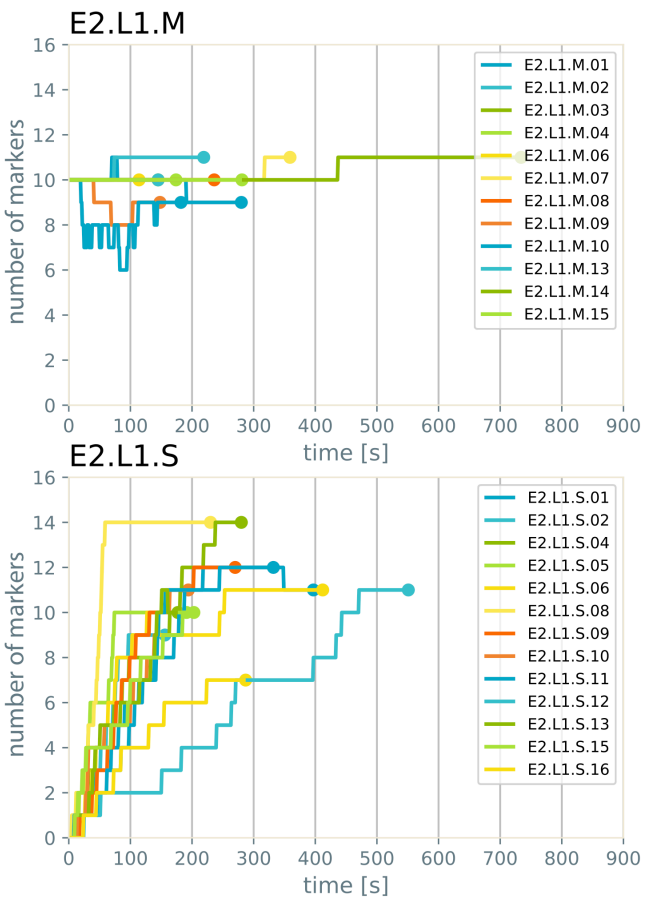

ration process, in which the number of rooms was reduced at the beginning to add and subtract rooms in the next steps in order to find the optimal minimum number of rooms. The second one is an example of the complete removal of the proposed initial design solution for independent configuration from scratch, as it is the case in the configuration in the $S$ mode.

The graphs allow also us to observe the difference in the rate of reaching the final number of rooms in the $\mathrm{S}$ mode. The steepness of the curve indicates the speed of configuration expressing the ease of use of the developed tool and its supporting role in visualizing solutions that the user already had in mind. During the experiment it was observed that some of the participants spoke out loud about their next steps, which wold allow them to achieve the desired solution.
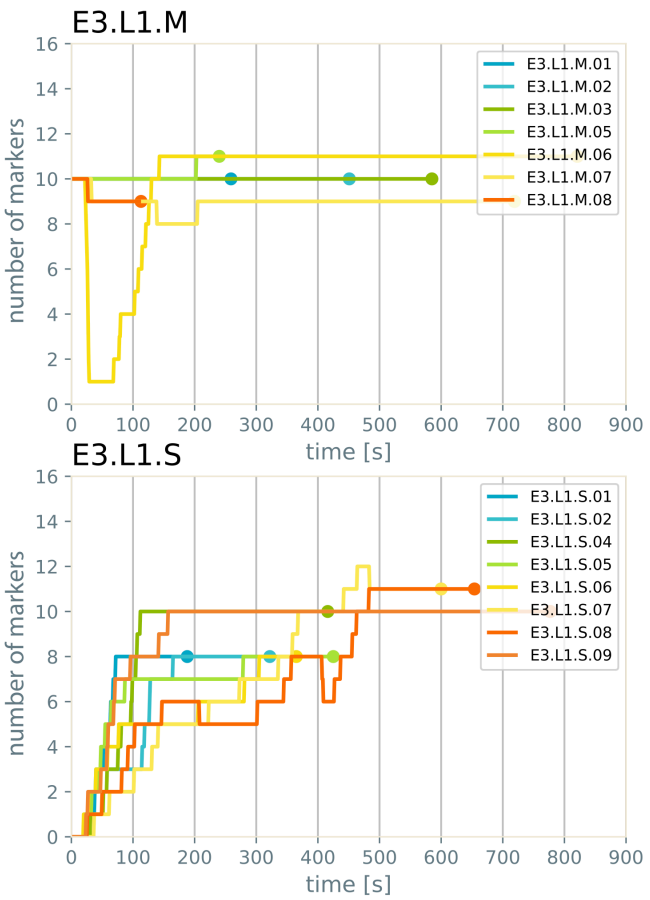

Participants were asked in the questionnaire to assess the usefulness of digital tools in supporting the participatory design of houses. All of participants confirmed "that tools like HOPLA could help in searching and finding housing design solutions fulfilling expectations". Additionally, usefulness was assessed using a five-point Likert scale. The median of the collected rating for the experiments conducted in the USA and in Poland were 5 (very useful) and 4, respectively. Mann-Whitney W-test did not reveal a statistically significant difference between the medians at the 95,0\% confidence level (Pvalue $=0,182752$ ). Obtained results indicate the demand from an unprofessional user for tools enabling computer-assisted co-design in the area of designing single-family houses.
Figure 5

Comparison of the number of displayed rooms during the configuration process. On the left, diagrams for the experiment conducted in the USA, and on the right in Poland. At the top, are the diagrams for the configuration in the $M$ mode, and at the bottom in the $S$ mode. 


\section{Users Customization Expectations}

Figure 6

Answers to the question: when customizing a design of your own house would you prefer to do it on your own using tool similar to HOPLA or be assisted by an architect?

Figure 7

Answers to the question: when planning your own house which option would you choose?

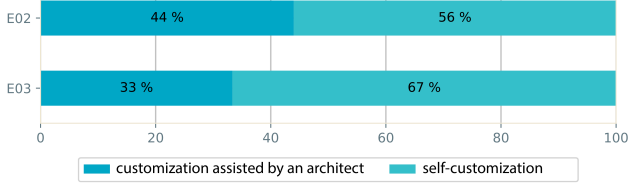

The preferences of participants regarding the method for obtaining a single-family home design, as well as information about the expectations of the respondents regarding the functionality of digital tools to support the customization of house designs were examined. The respondents were asked whether having the ability to configure the house design (using a tool like HOPLA) would they choose selfcustomization or customization assisted by a specialist/architect. In the case of both experiments, more than half of the respondents replied that they would prefer to configure the house themselves (56\% and $67 \%$ respectively for E02 and E03) (Figure 6).

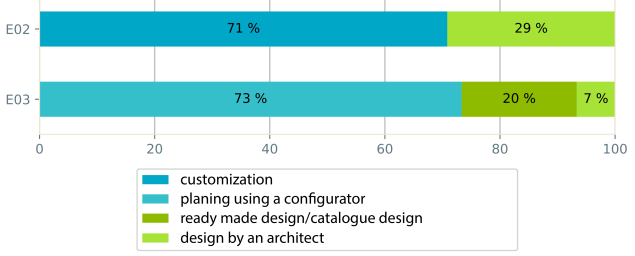

In addition, the respondents were also asked about the method of obtaining a house project. In the case of surveys conducted in the USA, the respondents had the choice between "customization" and "design by an architect." In the case of surveys conducted in Poland, the responses were adapted to local methods and included: "planning with the use of a configurator," "a ready / typical design from the catalog," and "an individual project (on request) by an architect." $71 \%$ of the participants in the experiment conducted in the US replied that they would choose customization instead of design made by an architect (Figure 7). In the experiment carried out in Poland,
$73 \%$ declared that they would choose to use a configurator, while only $7 \%$ would choose an individual project created by an architect.

As part of the survey, preferences regarding desirable functionalities of house design configurator were also collected. The participants were asked to order from the least important to the most important 10 listed functionalities of such a tool. The results of the survey for this issue for the experiments conducted in the USA and in Poland are shown in Figure 8.

For the research conducted in the USA, the Kruskal-Wallis test revealed a statistically significant difference amongst the rank medians of the listed customization possibilities at the $95,0 \%$ confidence level $(P$-value $=0,000072$ ). Pairwise comparison between the average ranks of the listed customization possibilities using the Bonferroni procedure, revealed a statistically significant difference at the $95,0 \%$ confidence level between "building layout customization" (median rank 8 out of 10-the most important) and all the other possibilities except for "site plan customization," "building technologies selection" and "building form modification."

In a comparison to the research conducted in Poland the Kruskal-Wallis test revealed statistical significant difference amongst the rank medians ( $P$ value $=0,000001$ ). Pairwise comparison between the average ranks of the listed customization possibilities using the Bonferroni procedure, revealed a statistically significant difference also between "building layout customization" (median rank 9 out of 10the most important) and "kitchen and wet rooms finishing material selection," "kitchen and wet rooms appliance selection," "furnishing selection," "exterior finishing materials selection" and "facade customization".

Results showed that there was no significant impact of cultural differences on the perception of the functionality of digital tools to support the co-design of single-family houses. Participants in both the experiments conducted in the USA and in Poland ranked the highest "building layout customization" 

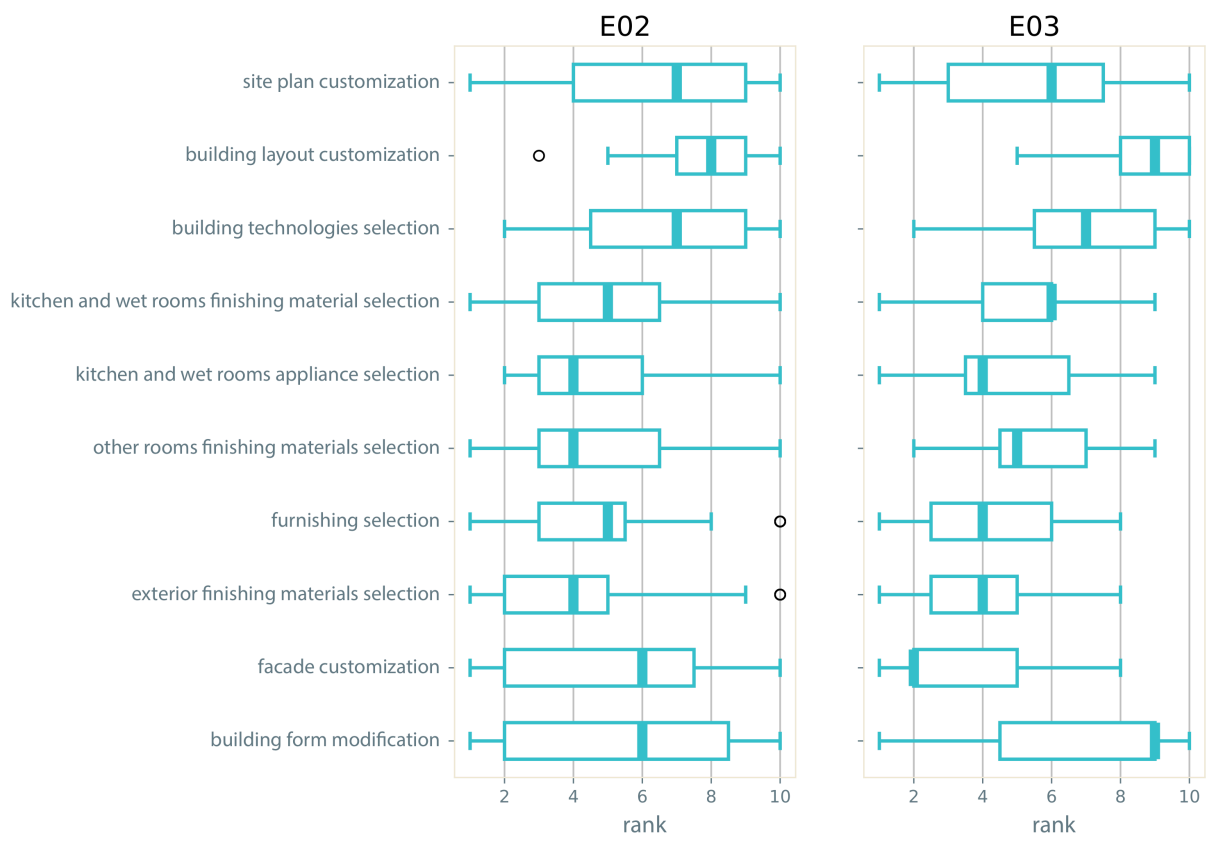

Figure 8

The result of the survey. Box plots of ranks of house design customization possibilities [1-less important, 10-the most important].

On the left, a summary for research conducted in the USA, and on the right in Poland.

for which comparison of medians using MannWhitney W-test showed that there could be some significant difference ( $P$-value $=0,0410248$ ), but after removing the outliers rank (rank 3 in E02), the test showed that there is not a statistically significant difference between the medians ( $P$-value $=0,0590178$ ). Additionally, there was not a significant difference between E02 and E03 medians of the customization possibilities that were ranked as the lowest. The least important functional feature was: "exterior finishing materials selection" ( $P$-value $=0,735932$ of comparison between E02 and E03 medians), "kitchen and wet rooms finishing material selection" ( $P$-value $=0,747266)$, "kitchen and wet rooms appliance selection" (P-value $=0,884028)$, "other rooms finishing materials selection" (P-value $=0,130371)$, "furnishing selection" (P-value $=0,558723$ ) and "facade customization" (P-value=0,183943). These features correspond precisely to the type of functionalities of- fered by current online house configurators.

Results also showed that for tool users in both experiments the most important feature was to provide information about the estimated building cost (Figure 9). Comparison of medians of this feature using Mann-Whitney W-test showed that there is not a statistically significant difference between the ranking of this feature by participants of the experiment conducted in Poland and the USA ( $P$-value $=0,225766$ ). Analysis of the least important customization outcomes showed some differences between expectations of the participants in both experiments. Pairwise comparison, for the E02 experiment, between the average ranks of the listed customization possibilities using the Bonferroni procedure, revealed a statistically significant difference between "estimated building costs" and two features: "possibility to order" and "specification of the selected choices". Additionally, pairwise comparison showed also that 
Figure 9

The result of the survey. Box plots of ranks of house

design

customization outcomes [1-less important, 10-the most important].

On the left, a summary for research conducted in the USA, on the right in Poland.

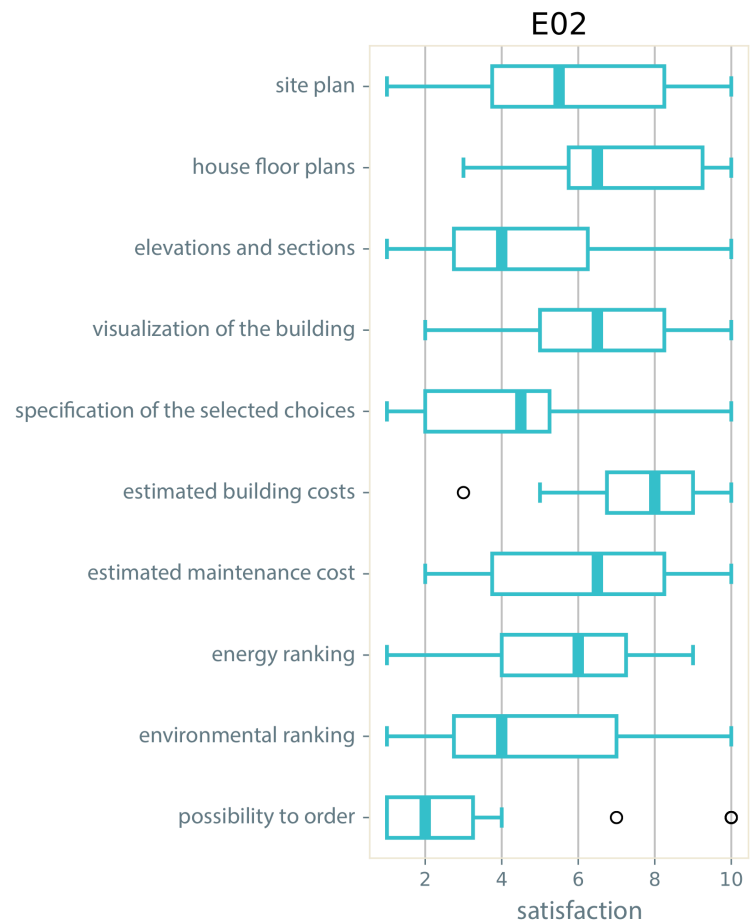

medians of these two features were significantly different from the median rank of "estimated building costs."

Analogous comparison performed for the results of the E03 experiment showed significant differences between "estimated building costs" and two features: "environmental ranking" and "possibility to order." "Environmental ranking" received the lowest median rank pairwise comparison and displayed also a significant difference between its median and the median rank of "estimated maintenance cost" and "house floor plans".

\section{CONCLUSIONS}

The conducted research confirmed the usefulness of the proposed computer tool for assisting in the cus- tomization of two-bay house designs. Usability tests showed that the proposed tool allows its users to find a solution that meets their expectations within a limited time frame. The proposed configuration process allowed all participants in the experiment to find design solutions in less than 15 minutes. All participants confirmed that tools such as HOPLA could help in searching for and finding design solutions that meet the expectations of future residents.

Asking users to modify a design solution confronts them with a design that must be evaluated, while enabling users to configure design solutions from scratch encourage them to first imagine their future home and then configured and visualized with the help of the tool. Also, while increasing the flexibility of the tool can increase users' satisfaction by 
permitting them to fulfill all their expectations, it is more challenging for the developers of the design system to guarantee the quality of design outcomes and compliance with desired design principles.

Most participants in both experiments declared that they would prefer to use a configurator for the design rather than other forms of obtaining a singlefamily house design, including asking an architect to design it. Additionally, more than half of the participants in both experiments stated that they would prefer to configure a design solution themselves rather than have the assistance of a specialist.

Research results did not reveal a significant impact of cultural differences on the expectations of non-expert users towards computer-assisted codesign of single-family houses but did show that these expectations are different from the currently available market offer. While for participants in both experiments the most important feature in the customization of houses was the possibility of modifying house layouts, the least important features were furniture, interior and exterior furnishings materials selection, which are currently offered to them by the construction market. Expectations on the results of configuration were similar in Poland and in the USA, where information about building cost was ranked as the first.

\section{REFERENCES}

Beirão, J. and Duarte, J. P. 2018, 'Generic Grammars for Design Domains', AIEDAM - Artificial Intelligence in Engineering Design and Manufacturing, 32, pp. 225239

Duarte, J.P. 2001, Customizing mass housing: a discursive grammar for Siza's Malagueira houses., Ph.D. Thesis, Massachusetts Institute of Technology

Huang, J. and Krawczyk, R. 2004 'i _ Prefab Home Customizing Prefabricated Houses by Internet-Aided Design', Proceedings of eCAADe 24, pp. 690-698

Khalili-Araghi, S. and Kolarevic, B. 2016, 'Development of a framework for dimensional customization system: A novel method for customer participation', Journal of Building Engineering, 5, pp. 231-238

Kwiecinski, K. and Markusiewicz, J. 2018 'HOPLA - Interfacing Automation for Mass-customization', Proceed- ings of eCAADe 36 , pp. 159-168

McLeish, T. J. 2003, A platform for consumer driven participative design of open (source) buildings, Master's Thesis, Massachusetts Institute of Technology

Mohamed, B. 2013, A Digital Platform for Mass Customization of Housing, Ph.D. Thesis, McGill University 